\title{
Chair Design Affects How Older Adults Rise from a Chair
}

\author{
Neil B. Alexander, MD, ${ }^{\dagger}$ Daniel J. Koester, MFA, ${ }^{\dagger}$ and Julie A. Grunawalt, MS, RNC ${ }^{\dagger}$
}

OBJECTIVE: To determine how modifications of key chair design aspects, such as seat height, posterior seat tilt, backrest recline, seat compressibility, and armrest placement, affect how older adults rise from a chair and the seating comfort they experience.

DESIGN: Cross-sectional comparison

SETTING: Congregate housing facility and university laboratory

SUBJECTS: Two groups of volunteers, Old ( $\mathrm{n}=29$, mean age 84) and Young ( $n=21$, mean age 23).

MEASUREMENTS: Analysis of time to rise, body motion (determined by use of digitized videotaping), and selfreported difficulty when subjects rose from a variety of controlled chair settings thought to represent important chair design specifications encountered by older adults. Subjects also reported their comfort while being seated in these settings.

RESULTS: Lowered seat height, increased posterior seat tilt and backrest recline, and perhaps increased seat compressibility cause increased time to rise, increased body motion, and increased self-reported ratings of rise difficulty in both Young and Old groups. Under the most challenging conditions, the effect appears to be stronger in the Old than in the Young: a few Old were unable to rise, and the Old took disproportionately longer to rise and used disproportionately greater neck motion ( $P$ generally $<0.001$ ) compared with the Young. Arm rest placement did not alter rise performance or ratings significantly. The conditions in which rise difficulty increases or decreases do not correspond exactly to conditions in which comfort increases or decreases. Some aspects that increase rise difficulty, such as tilt/recline and seat compressibility, may also increase comfort.

CONCLUSIONS: Aspects of chair design such as lowered seat height, increased posterior seat tilt, increased back recline, and increased compressibility interfere with chair egress in older adults. While decreasing ease of egress, however, these same factors may increase seating comfort. Furniture designers and manufacturers must find a balance between degree of sitting comfort, ease of egress, and the degree

From the "Division of Geriatric Medicine, Department of Internal Medicine, University of Michigan; and the ${ }^{\dagger}$ Geriatric Research, Education, and Clinical Center, Department of Veterans Affairs Medical Center, Ann Arbor, Michigan.

This material is based on work supported by the Rehabilitation Research and Development Service, Department of Veterans Affairs, and by National Institutes on Aging Grant AG 00519. Presented, in part, at the American Geriatrics Society Annual Meeting, November 1993.

Address correspondence and reprint requests to Neil Alexander, MD, Division of Geriatric Medicine, 300 North Ingalls Building, Ann Arbor, MI 48109. to which the seating device facilitates functional independence, particularly to meet the needs of disabled older adults. J Am Geriatr Soc 44:356-362, 1996.

$\mathrm{D}^{\mathrm{s}}$ ifficulty in rising from a chair affects up to 2 million $(8 \%)$ of noninstitutionalized persons aged 65 and older., Others have studied the biomechanics of how older adults rise from a chair, ${ }^{3-6}$ but relatively few have considered how aspects of chair design might affect rising in older adults. Many older adults depend on certain key features of the chair, such as the armrest, for assistance in rising. ${ }^{7}$ At least three studies ${ }^{8-10}$ suggest that raising the seat height decreases rise difficulty. In a comparison of two commercially available chairs, ${ }^{11}$ older women had more difficulty rising from the chair with greater posterior tilt and backrest recline and shorter armrest height. Other common design aspects that affect rising from a chair, such as armrest placement and seat compressibility, are rarely studied empirically.

The most important factor that many older adults, particularly those with osteoarthritis, consider when choosing a chair is the ease with which they can rise from the chair. ${ }^{7}$ Yet, few studies contrast the effect of chair design on seating comfort with the effect of chair design on rising, i.e., the relationship between comfort and ease of egress. There are published chair design criteria that purport to improve safety, comfort, and ease of rising for older adults. ${ }^{12-14}$ Unfortunately, there is limited empirical data to support these design choices, particularly data regarding how design alterations affect performance.

Adjustable evaluation chairs have been used in controlled laboratory settings to develop chair design criteria for comfort while performing specialized tasks such as surgery. ${ }^{15}$ This more controlled methodology allows a range of design criteria to be simulated in order to determine their effect on user performance. Similarly, we used an adjustable laboratory chair to simulate many of the chair configurations encountered daily by older adults. We also tested the effect of each design factor individually, rather than with multiple factors at once, and provided a more controlled test situation by standardizing seat height as a function of body size. We hypothesized that each of the following key chair design settings - lowered seat height, increased posterior seat tilt, increased backrest recline, increased seat compressibility, and lowered or more posteriorly placed arm rests - increases chair rise difficulty in older adults. This difficulty would be suggested by increased duration of rise time, increased body motion used to rise, and subjective ratings of greater rise difficulty, when compared with a condition with more stan- 
dardized chair design features. Furthermore, we hypothesized that chair settings that result in greater subjective rise difficulty might also be rated as more comfortable.

\section{METHODS}

\section{Subjects}

Twenty-one Young (mean age 23, range 20-28) and 29 Old (mean age 84, range 73-93) adults volunteered to participate in this study. All Young were recruited from a group of undergraduate University of Michigan students. The Old were recruited from a list of residents in a local congregate housing facility who had previously indicated their interest in participating in research projects. All of the Old lived independently in their own apartments and were able to rise independently from a standard chair with or without the use of their arms. All of the Old were able to walk independently, although five $(17 \%)$ used canes and three $(11 \%)$ used walkers, at least occasionally. Old subjects with dementia (Folstein Mini-Mental Status score $<23$ ), severe musculoskeletal deformities (such as post-polio syndrome), and acute illness (such as cardiopulmonary instability) were excluded ( $\mathrm{n}=3$ total).

Some of the Old were found to have cardiovascular, musculoskeletal, and/or neurological abnormalities on standardized history and physical examination conducted by a trained nurse practitioner. The most common diagnoses and symptoms thought to be present (in percent of the group) were: cardiovascular-related diagnosis, primarily hypertension $(65 \%)$; hearing loss $(58 \%)$; osteoarthritis, primarily of the lower extremity and occasionally symptomatic (42\%); symptomatic feet problems $(27 \%)$; and a history of a hip operation $(23 \%)$. All but two subjects reported being righthanded. On examination, nearly all subjects exhibited asymmetry or reduction of a lower extremity reflex, approximately half had reductions in either upper extremity or lower extremity strength, and approximately one-third had reductions in either vibration sense, position sense, or sharp-dull discrimination.

\section{Equipment, Data Acquisition, and Protocol}

Subjects rose from an adjustable laboratory chair in which five key design aspects were varied: vertical seat height, anteroposterior seat tilt, posterior back rest recline, vertical and horizontal location of the arm rests, and, with the addition of a 4-inch foam pad, seat compressibility. The starting reference seating configuration was standardized (standard condition) to include placement of the seat at $100 \%$ floor to knee height $(100 \% \mathrm{FKH})$, the ankle at 90 degrees, and the seat back rest at 5 degrees recline from vertical i.e., 95 degrees. The seat had no anteroposterior tilt but had 1/2-inch padding (Medium Density Sunmate, Sunmate Design Systems, Leizester $\mathrm{NC}$ ) covered by vinyl. The seat was $51 \mathrm{~cm}$ wide mediolaterally and $40 \mathrm{~cm}$ long anteroposteriorly (in two detachable $20-\mathrm{cm}$ sections, see below). In addition, the armrest was placed so that the arm was vertical and the elbow was at 90 degrees.

Subjects rose from the chair under 10 conditions in the following order (see Table 1): Standard (100\% FKH); $120 \%$ (seat at $120 \% \mathrm{FKH}$ ); $140 \%$ (seat at $140 \%$ FKH); ArmrestLow (armrests lowered to one-half the original standard vertical distance between the elbow and the seat, also abbreviated as AR-Low); Armrest Forward (armrests moved forward 1.5 times the length of the elbow tip to the fourth metacarpal head, also abbreviated as AR-Fwd); Foam (sofa cushion foam placed on top of $80 \%$ FKH); $80 \%$ (seat at $80 \%$ FKH); 60\% (seat at $60 \%$ FKH); 10/105 (seat tilt 10 degrees anteroposteriorly and backrest recline 105 degrees posteriorly); and 20/115 (seat tilt 20 degrees anteroposteriorly and backrest recline 115 degrees posteriorly). The simulated sofa cushion, HR 65 Polyurethane Foam, was a medium-hard upholstery foam (American Excelsior, Westland MI) with a density that required 65 pounds to compress to threequarters of its size. The foam was 4 inches thick (as per many sofa cushions) and cut to the width and length dimensions of the seat described above.

These seating conditions were chosen to represent a number of seating situations encountered regularly by older adults. The 10/105 was supposed to represent a lounge chair or anti-thrust seat that might be used in a "geri-chair," and the $20 / 115$ was thought to represent a semi-recliner position. The seat heights $140 \%, 120 \%, 100 \%, 80 \%$, and $60 \%$ were chosen to simulate, respectively, a bar stool or sit-stand position, a high standard chair, a standard chair, a low standard chair, and a foot stool or ottoman. Given the floor to knee heights in both groups (mean $44 \mathrm{~cm}$, range 39-55 $\mathrm{cm}$ ), means for the experimental chair heights used for the $140 \%, 120 \%, 100 \%, 80 \%$, and $60 \%$ conditions were 62 ,

Table 1. Chair Rise Conditions

\begin{tabular}{|c|c|c|c|c|c|c|}
\hline $\begin{array}{c}\text { Order } \\
\text { Performed }\end{array}$ & Condition Title & $\begin{array}{c}\text { Seat Height } \\
\text { (\% floor-knee } \\
\text { height) }\end{array}$ & $\begin{array}{l}\text { Armrest } \\
\text { Position }\end{array}$ & $\begin{array}{c}\text { Seat } \\
\text { Foam/Tilt }\end{array}$ & $\begin{array}{l}\text { Backrest } \\
\text { Recline } \\
\text { (degrees) }\end{array}$ & Seating Simulated \\
\hline 1 & Reference & 100 & Standard & None & 95 & Standard Chair \\
\hline 2 & $120 \%$ & 120 & Standard & None & 95 & High Standard Chair \\
\hline 3 & $140 \%$ & 140 & Standard & None & 95 & Bar Stool \\
\hline 4 & Armrest-Low & 100 & Low & None & 95 & \\
\hline 5 & Armrest-Forward & 100 & Forward & None & 95 & \\
\hline 6 & Foam & 80 & Standard & Foam & 95 & Sofa \\
\hline 7 & $80 \%$ & 80 & Standard & None & 95 & Low Standard Chair \\
\hline 8 & $60 \%$ & 60 & Standard & None & 95 & Foot Stool \\
\hline 9 & $10 / 105$ & 100 & Standard & 10 deg & 105 & Lounge Chair \\
\hline 10 & $20 / 115$ & 100 & Standard & 20 deg & 115 & Recliner \\
\hline
\end{tabular}


$53,44,35$, and $26 \mathrm{~cm}$, respectively (or $24,21,17,14$, and 10 inches, respectively).

In order to maintain the subjects' feet in contact with the floor at the required starting ankle configuration, the anteroposterior seat length was reduced from $40 \mathrm{~cm}$ to $20 \mathrm{~cm}$ for the $140 \%$ and $120 \%$ conditions only. For shorter persons, and particularly during $60 \%$, a platform was used underneath the feet to achieve a sufficiently lowered seat height. Also, the subjects' feet were placed on a board upon which was painted a 1-cm gradation grid for documentation of the maximum amount of posterior foot movement occurring during the rise.

Subjects were videotaped from their right side as they rose from the adjustable laboratory chair. Timing of the rise was thus accomplished through an inset timer. The rise was initiated by the onset of head motion and ended with the establishment of upright stance. In order to ascertain when the lift-off from the seat occurred, a seat switch was connected to a light underneath the seat. The light went out when the subject's thighs were no longer in contact with the seat. The time elapsed between motion onset to lift-off was designated as Phase 1 time, with total time taken at upright stance.

Target markers were placed on the skin overlying key body landmarks of the right side (lateral malleolus, lateral femoral condyle, greater trochanter, midway between the lateral femoral condyle and greater trochanter, lateral reflection of the acromion, and in front of the tragus) for subsequent calculation of body segment motion. Using a computer attached to a monitor, a frame grabber was used to capture the body position at lift-off. The captured image was digitized, and by connecting the markers on a computer screen, the angle of the neck, hip, and knee immediately before rise onset and at lift-off were calculated and written to a file. The change in angle from rise onset to lift-off (Phase 1 Angle Change) was then derived.

Although all of the subjects had controlled starting configurations, they were told to rise in a way they felt comfortable, using any motions necessary. Subjects performed two trials at each condition, with the first considered practice and the second used for data collection. During the rare occasions when a subject rocked on the seat, the data were collected from the last successful rise sequence. Essentially, no subject scooted completely forward to the edge of the chair before lift-off from the seat.

For each condition, subjects were asked to rate (1) how much difficulty they had in rising and (2) how comfortable each initial seating position was, in comparison with the $100 \%$ reference condition. A 5 -point scale was used, with a lower score representing less difficulty and more comfort than the reference condition. For the difficulty scores, 1 represented much less difficulty; 2 somewhat less difficulty, 3 no difference, 4 more difficulty, and 5 much more difficulty. For comfort scores, 1 represented much more comfortable, 2 somewhat more comfortable, 3 no difference, 4 somewhat less comfortable, and 5 much less comfortable. One additional $100 \%$ reference trial was performed after the $140 \%$, Armrest-forward, and $60 \%$ conditions in order to facilitate the difficulty and comfort rating comparisons.

\section{Data Analysis}

Four sets of comparisons were made, each between the initial reference condition $(100 \%)$ performance, and changes in height $(140 \%, 120 \%, 80 \%, 60 \%)$, armrest placement (armrest-low, armrest forward), compressibility (foam), and tilt/recline (10/105, 20/115). Mean Phase 1 and Total Rise time, Phase 1 angle, and maximum posterior foot movement were compared using repeated measures ANOVA. Median difficulty and discomfort ratings were compared using nonparametric Friedman and Mann Whitney $U$ tests.

\section{RESULTS}

\section{Rise Ability}

Two Old subjects were unable to complete the $60 \%$ condition rise, and one of these Old subjects was also unable to complete the 20/115 rise. These subjects could not be included in the height and the tilt/recline ANOVA but were included in the other comparisons below.

\section{Timing Data}

Mean Total Rise times appear in Figure 1. As expected, the Old generally take longer to rise in most of the conditions, particularly the seat height, recline/tilt, and foam conditions (between group $P$ generally $<.001$ for Total Time comparisons). The chair rise challenge increases with decreasing seat height, increasing tilt/recline, and with the addition of foam, i.e., as one moves from left to right in Figure 1 (condition effect $P$ generally $<.001)$. Whereas the Young take slightly longer to rise as the chair condition challenge increases, the Old take disproportionately longer to rise. Hence, the repeated measures condition $\times$ group effect becomes significant. The most striking effects occur with the change in seat height. As the height lowers from $140 \%$, particularly to the $80 \%$ and $60 \%$ levels, the rise time increases; this occurs most dramatically in the Old $(P<.001$ for condition $\times$ group Total Time comparisons). The same trend is noted in the tilt/recline conditions, particularly for $20 / 115(P<.001$ for condition $\times$ group effect). There is also an effect for foam in both between group and within condition comparisons $(P<$ $.005)$, with a borderline significant condition $\times$ group effect $(P=.06)$. Armrest placement does not appear to affect timing. Thus, based on the timing data, the Old are disproportionately challenged by lowered seat heights, increased seat tilt/recline, and perhaps by a compliant surface.

The mean Phase 1 rise times (data not displayed) range from 0.6 to 1.3 seconds with the highest values in both Young and Old for the $60 \%$ and $20 / 115$ conditions. The data tend to follow the trends above, although only the effect of low height $(P<.001)$ and increased tilt/recline $(P<.001)$ are statistically significant. There is a also disproportionate (condition $\times$ group) effect in the Old for increased tilt/recline $(P<$ $.01)$. For the $100 \%$ reference conditions performed after the $140 \%$, Armrest-forward, and $60 \%$ conditions, there is no significant increase in Total Rise time compared with the first $100 \%$ reference trial, suggesting that fatigue did not influence rise performance during later parts of the protocol.

\section{Angle Change Data}

Mean Phase 1 angle change from start to lift-off at the neck and hip are displayed in Figures 2 and 3. Overall, the Old flex their necks significantly more and hips significantly less in all four comparisons (between group comparisons at least $P<.005$ and generally $P<.001$ ). For both groups across each comparison, hip flexion increased significantly as the seat was lowered $(P<.001)$, when foam was added $(P<$ $.002)$, and with increasing tilt/recline $(P<.001)$. Neck flexion also increased as the seat was lowered $(P<.001)$, 


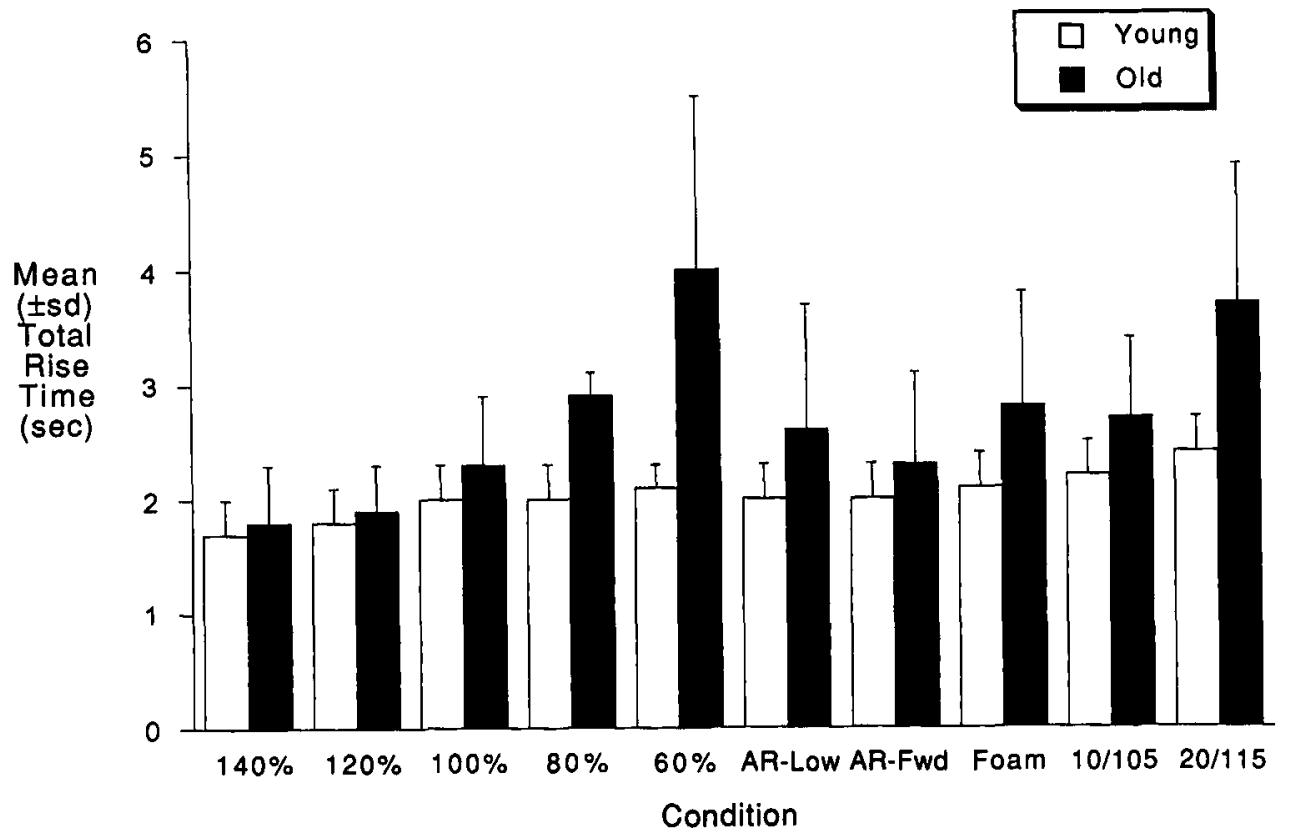

Figure 1. Mean Total Rise time (in seconds according to chair design condition. Condition abbreviations are as follows: corresponding to $140,120,100,80$, and $60 \%$ of floor to knee height; armrest -low (AR-Low) and armrest-forward (AR-Fwd); seat cushion (Foam); and seat tilt/backrest recline of 10/105 deg (10/105) and 20/115 deg (20/115). See Table 1 for further description of conditions. Error bars represent standard deviation.

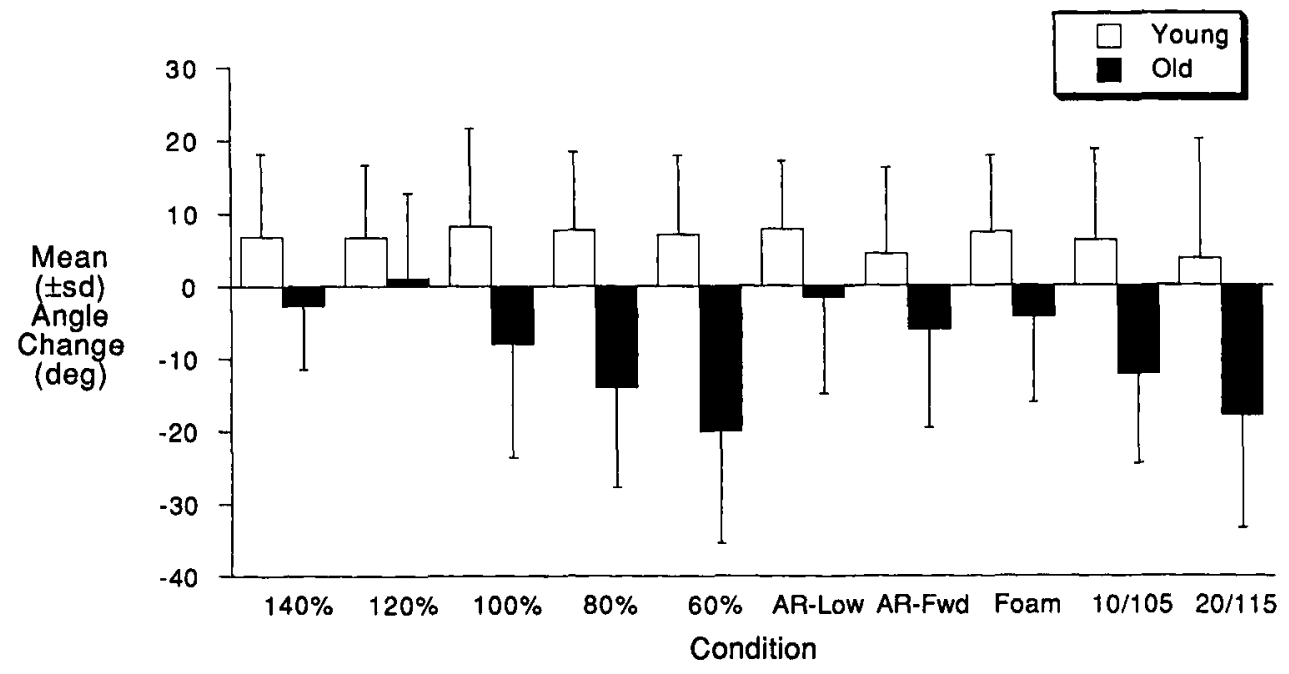

Figure 2. Mean Phase 1 angle change for neck motion. Positive changes denote neck extension, and negative changes denote neck flexion. See Figure 1 and Table 1 for explanation of conditions. Error bars represent standard deviation.

disproportionately so in the Old $(P<.001$ for condition $x$ group effect) and with increasing tilt/recline $(P<.005$ for condition $\times$ group effect). Mean angle changes at the knee, ranging from 0.2 to 2.4 degrees, are not statistically significant, and as with the neck flexion data, the variability is large and there is a tendency for both extension and flexion changes to be found in both groups. Similar to the timing data, the most marked joint motion changes are seen as the seat is lowered and tilt/recline increased, although only the Old are disproportionately affected in neck flexion as the seat is lowered.

\section{Foot Movement Data}

Mean maximum posterior foot movement (data not displayed) ranges from 1.5 to $3.7 \mathrm{~cm}$ in the Young and from
3.5 to $7.1 \mathrm{~cm}$ in the Old. Generally, the Young tend to move their feet less than the Old in preparation to rise, although the only significant between-group differences occur for the arm rest and tilt/recline conditions ( $\mathrm{R}$ foot only, $P<.05$ for both conditions). The most striking condition effect in both groups occurs in $140 \%$, where the mean foot movement (2.3 and 1.5 $\mathrm{cm}$ for Young, 3.5 and $3.9 \mathrm{~cm}$ for Old, $\mathrm{L}$ and $\mathrm{R}$ foot respectively) is 1 to $3 \mathrm{~cm}$ less than mean foot movement in most of the lower seat height conditions $(P<.01$ for $\mathrm{L}$ and $\mathrm{R}$ foot condition effect). As with the angle data, there was substantial variability in both groups across the various conditions.

\section{Subjective Difficulty and Comfort Ratings}

Median group ratings of difficulty in rising for a particular condition, compared with the $100 \%$ reference condition, 


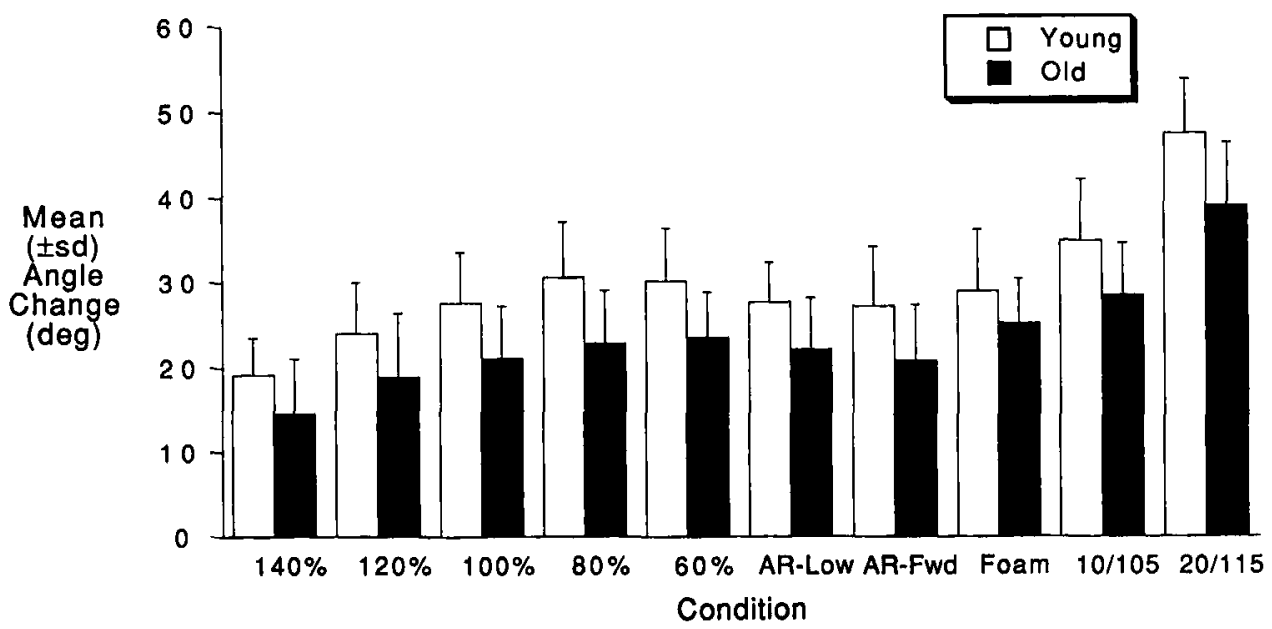

Figure 3. Mean Phase 1 angle change for hip flexion. See Figure 1 and Table 1 for explanation of conditions. Error bars represent standard deviation.

appear in Figure 4a. As the seat is lowered or placed into a tilt/recline position, the median score increases, reaching a level of 4 (somewhat more difficult) for tilt/recline and 5 (much more difficult) for $60 \%$ (Friedman $P<.001$ for both sets of conditions). Difficulty is decreased substantially as the height is increased (Friedman $P<.001$ ). Foam appears to increase difficulty (Mann-Whitney U $P<.001$ ), whereas
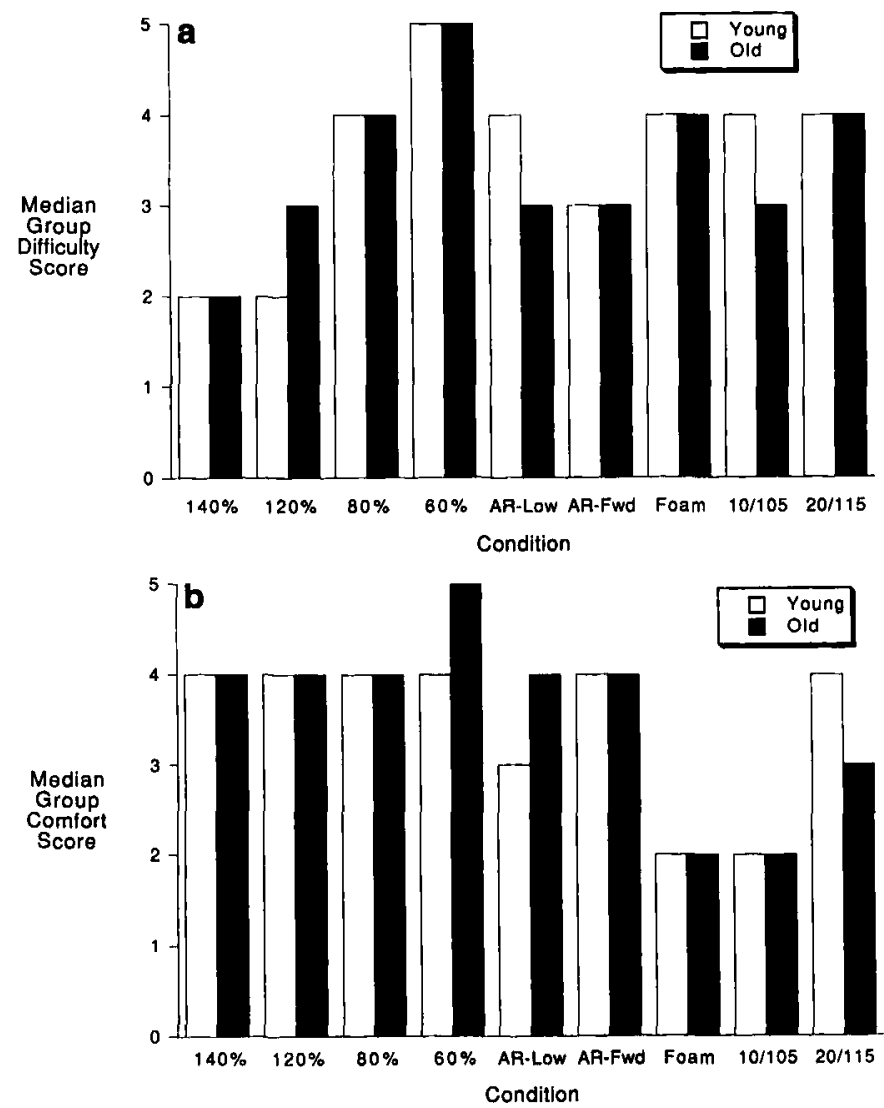

Figure 4. Median group self-reported ratings according to chair design condition compared with $100 \%$ reference condition. a. Difficulty experienced while rising during condition; b. Comfort experienced while seated in condition. See Figure 1 and Table 1 for explanation of conditions. armrest position generally does not. In contrast, when compared with the reference $100 \%$ condition, the foam and $10 / 105$ tilt/recline positions are rated as more comfortable (median scores of 2, Mann Whitney U $P<.005$ for both, see Figure 4b). All height changes are viewed as significantly less comfortable (Friedman $P<.001$ ). Thus, the conditions in which rise difficulty is increased or decreased do not correspond exactly to conditions in which comfort is increased or decreased. Increased seat height may decrease rise difficulty, but it may decrease comfort. Foam and increased tilt/recline may increase rise difficulty, but they may be viewed as more comfortable.

\section{DISCUSSION}

Although it is clear that certain aspects of chair design, such as seat height, affect ease of rising in older adults, the relative influence of these aspects on seating comfort and ease of egress are unclear. Timing, body motion, and self-report difficulty data from the present study suggest that aspects such as seat height, tilt/recline, and seat compressibility increase egress difficulty. On the other hand, some aspects that increase rise difficulty, such as tilt/recline and seat compressibility, may also increase comfort.

Seat height is frequently mentioned as the most important chair design factor influencing egress. ${ }^{7}$ Increasing seat height decreases the joint range of motion and strength required by the hips and knees to rise. ${ }^{16}$ Yet, seating that is too high can compromise peripheral circulation and, particularly when the feet are not in contact with the floor, postural stability. ${ }^{14,17,18}$ These factors may explain why the subjects in the present study were not necessarily more comfortable at the higher seat heights. A number of investigators recommend 17 inches as the optimal seat height, when measured from the floor. ${ }^{8,14,19}$ Weiner et al. ${ }^{10}$ recently suggested that the optimum seat height for ease of egress should be $120 \%$ of leg length for modestly impaired subjects, corresponding to a range of 18 to 22 inches for the range of leg lengths in their study. Weiner et al. ${ }^{10}$ also found that common seat heights for chairs and couches in the community are lower than the optimum seat height, ranging from 15 to 17 inches. These seat heights are within the range of the mean heights tested in the present study, including means of 14 inches $(80 \%), 17$ inches $(100 \%)$, and 21 inches $(120 \%)$. 
A number of investigators ${ }^{12,17,18}$ have made recommendations regarding seating for older adults, particularly for those with arthritis and low back pain, and many of these recommendations are confirmed by the present study. They suggest that egress is more difficult from seats that are low and soft, seats that are too deep (such that the occupant must scoot forward) and that have too much rake (tilt) angle (recognizing that too little rake angle may allow the occupant to slide forward). Other important factors to consider include the slope of the backrest (sufficient to allow leaning back without inhibiting egress). ${ }^{12,14}$ Holden et al. ${ }^{12}$ discuss recommendations for optimal seat tilt of up to 10 degrees and backrest recline up to 105 degrees, two settings that were tested in the present study and found to aid comfort but perhaps impede egress. Furthermore, the chair must have enough clearance under the seat for the occupant to be able to place the feet under the front edge of the seat. Placing the feet backwards underneath the upper body segments assists the rise because it enhances anteroposterior stability at liftoff. ${ }^{6}$ In the present study, even with the clearance available for posterior feet placement, maximum posterior foot movement does not differ dramatically between conditions except at the $140 \%$ seat height.

Armrests that are high and come forward to the seat's front edge facilitate egress by helping the occupant move forward to the edge of the seat and by assisting the occupant in rising and maintaining stability while rising. ${ }^{12}$ Finlay $^{8}$ found armrest height to be an important factor, with subjects able to rise at armrest heights of 10 inches (vs 7 inches) above seat height. Our reference armrest position, with the elbow at 90 degrees, might be viewed as satisfactory, and although the low armrests should have hampered rising, the forward armrests should have facilitated rising. Nevertheless, differences in timing, body motion, and subjective evaluations of rise difficulty and comfort were small between the three armrest conditions. At least for the young and old adults included in this study, armrest placement was not as important a predictor of rise performance as the seat-related modifications.

Modifications in chair design may be necessary in some mobility-impaired older adults. Seat height, for example, can be modified by supplementing chair leg length. ${ }^{10,19}$ Sometimes a foot rest is recommended in order to elevate the thighs and support the feet when seat heights are too high, ${ }^{9}$ although the foot rests may also become a hazard during rising. ${ }^{12,18}$ Unfortunately, because of differences in body anthropometry, seat heights may need to be individualized, ${ }^{18}$ and this may be impractical in an institutional and, perhaps, even a community setting. ${ }^{12}$

Seating comfort on a variety of chairs has been sampled in many settings, ${ }^{20-25}$ often for people with back pain ${ }^{26,27}$ and arthritis. ${ }^{28}$ Older adults are frequently uncomfortable even on standard chairs, and they often use cushions of varying size and compliance that may augment comfort but impede egress. ${ }^{7}$ In addition to the present study, others note that comfort may conflict with ease of egress. ${ }^{29}$ Chairs with elevated seats, such as those that place people in a sit-stand position (similar to $140 \%$ in the present study) are rated as less comfortable than conventional seating. ${ }^{26}$ Note that reported comfort may vary not just regarding the relationship of the chair fit to the subject's anatomy, as we have proposed, but also in regard to the concurrent task performed by the subject. ${ }^{21}$ Clearly, particularly for disabled older adults, furniture designers and manufacturers must find a balance between degree of sitting comfort, ease of egress, and the degree to which the seating device facilitates functional independence.

Ease of egress and comfort may not be the only factors influencing how older adults choose their seating. Others cite ease of ingress and the stability of the chair so that the chair does not tip during rising or when it is used for support when walking around a room..$^{7,12-14}$ Ultimately, price and the aesthetic appeal may be as important as the above factors. ${ }^{18}$ Thus, when making recommendations regarding appropriate seating for older adults, clinicians should consider the degree of patient disability, ease of ingress and egress, sitting comfort, need for concurrent task performance while seated, chair stability, price, and aesthetics.

\section{ACKNOWLEDGMENTS}

The authors acknowledge the assistance of Becky Luebke, Michael Felkey, and Cristen Stirrup in data acquisition, processing, and analysis.

\section{REFERENCES}

1. National Center for Health Statistics. Dawson D, Hendershot G, Fulton J et al. Aging in the Eighties. Functional limitations of Individuals Age 6.5 and Over. DHHS Pub. 87-12.50. Public Health Service, 1987.

2. Leon J, Lair T. Functional Status of the Non-institutionalized Elderly: Fstimates of ADL and IADL. Difficulties. DHHS Pub. PHS 90-3462. Public Health Service, 1990.

3. Alexander NB, Schultz AB, Warwick DN. Rising from a chair: Effects of age and functional ability on performance biomechanics. J Gerontol 1991;46:M91-98.

4. Ikeda ER, Shenkman ML, Riley PO et al. Influence of age on dynamics of rising from a chair, Phys Iher 1991;71:473-481.

5. Millington PJ, Myklebust BM, Shambes GM. Biomechanical analysis of sitto-stand motion in elderly persons. Arch Phys Med Rehabil 1992;73:609617

6. Schultz AB, Alexander NB, Ashton-Miller JA. Biomechanical analyses of rising from a chair. J Biomech 1992;25:1383-1391.

7. Munton IS, Ellis MI, Chamberłain MA et al. An investigation into the problems of easy chairs used by the arthritic and the clderly. Rheumatol Rehabil 1981;20:164-17.3.

8. Finlay OE, Bayles TB, Rosen $C$ et al. Effects of chair design, age, and cognitive status on mobility. Age Ageing 198.3;12:329-335.

9. Burdett RG, Habasevich R, Pisciotta J et al. Biomechanical comparison of rising from two types of chairs. Phys Ther 198.5;65:1177-1183.

10. Weiner DK, Long R, Hughes MA et al. When older adults face the chair-rise challenge. J Am Cicriatt Soc 1993;41:6-10.

11. Wheeler J, Woodard C, Ucovich RL et al. Rising from a chair: Influence of age and chair design. Phys Ther 1985;65:22-26.

12. Holden JM, Fernie G, Lunau K. Chairs for the elderly-Design considerations. Appl Ergon 1988; 19:281-288.

13. Finlay OE. Rehabilitation chair. Physiotherapy 1981;67:207.

14. Christenson MA. Aging in the Designed Environment: Chair Design and Selection for Older Adults. Binghamron, NY: Haworth Press, 1990, pp 67-85.

15. Congleton JJ, Ayoub MM, Smith JL. The design and evaluation of the neutral posture chair for surgeons. Hum Factors 1985;27:589-600.

16. Rodosky MW, Andriacchi TP. Andersson (;BJ. The influence of chair height on lower limb mechanics during rise. J Orthop Res 1989;7:266-271.

17. Chamberlain MA, Munton J. Designing chairs for the disabled arthritic. $\mathrm{Br}$ J Rheumatol 1984;23:304-308.

18. Sweeney GM, Clarkc AK. Selecting casy chairs for people with arthritis and low back pain. Physiotherapy 1991;77:509-511.

19. Hyland PA. Chair assessment and three methods of raising chairs. Br J Oc cup Ther 1979;42:269-272.

20. Jones JC. Methods and results of seating research. Ergonomics $1969 ; 12: 171-181$.

21. Shackel B, Chidsey KD, Shipley P. The assessment of chair comfort. Ergonomics 1969;12:269-306. 
22. Barkla DM. Chair angles, duration of sitting, and comfort ratings. Ergonomics 1964;7:297-304.

23. Grandjean $E$, Hunting W, Wotzka $G$ et al. An ergonomic investigation of multipurpose chairs. Hum Factors 1973;15:247-253.

24. Grandjean E, Hunting W. Ergonomics of posture-Review of various probIcms of standing and sitting posture. Appl Ergon 1977;8:135-140.

25. Le Carpentier EF. Easy chair dimensions for comfort-A subjective approach. Ergonomics 1969;12:328-337.
26. Michel DP, Helander MG. Effects of two types of chairs on stature change and comfort for individuals with healthy and herniated discs. Ergonomics 1994;37:12.31-1244.

27. Grandjean E, Boni A, Kretzschmar H. The development of a rest chair profile for healthy and notalgic pcople. Ergonomics 1969;12:307-315.

28. Atherton JA, Chatfield J, Clarke AK et al. Static chairs for the arthritic. Br J Occup Ther 1980;43:366-367.

29. Shipley P. Chair comfort for the elderly and infirm. Nursing $1980 ; 20: 858-860$. 